\title{
Force chains in monodisperse spherical particle assemblies: Three-dimensional measurements using neutrons
}

\author{
C. M. Wensrich, ${ }^{1,}{ }^{*}$ E. H. Kisi, ${ }^{1}$ V. Luzin, ${ }^{2}$ U. Garbe, ${ }^{2}$ O. Kirstein,,${ }^{1,3}$ A. L. Smith, ${ }^{1}$ and J. F. Zhang ${ }^{1}$ \\ ${ }^{1}$ The University of Newcastle, University Drive, Callaghan NSW 2308, Australia \\ ${ }^{2}$ Bragg Institute, Australian Nuclear Science and Technology Organisation, Locked Bag 2001, Kirrawee NSW 2232, Australia \\ ${ }^{3}$ European Spallation Source, Box 176, S-221 00 Lund, Sweden
}

(Received 30 July 2014; published 3 October 2014)

\begin{abstract}
The full triaxial stress state within individual particles in a monodisperse spherical granular assembly has been measured. This was made possible by neutron imaging and computed tomography combined with neutron diffraction strain measurement techniques and associated stress reconstruction. The assembly in question consists of 549 precision steel ball bearings under an applied axial load of $85 \mathrm{MPa}$ in a cylindrical die. Clear evidence of force chains was observed in terms of both the shape of the probability distribution function for normal stresses and the network formed by highly loaded particles. An extensive analysis of the source and magnitude of uncertainty in these measurements is also presented.
\end{abstract}

DOI: 10.1103/PhysRevE.90.042203

PACS number(s): 45.70.-n, 61.05.fm, 07.10.Lw

\section{INTRODUCTION AND BACKGROUND}

The penetrating power of neutrons has long been used to study the internal structure of solids. For some time, neutrons have also been used to probe strain and stress distributions within solid engineering materials (e.g., see Refs. [1-4]). Recent work in this area has extended these routine techniques to applications within granular materials [5,6]. This has provided the opportunity to measure and map out fully resolved triaxial bulk stress distributions within granular assemblies undergoing high-stress die compaction processes $[7,8]$. The information gained through these techniques provides a wealth of understanding on the quasicontinuum (or bulk) behavior of granular materials. In this paper we now turn our attention to using the same approach to understand the discrete behavior of these systems.

Granular materials are a unique form of matter that exhibit complex load sharing behavior at length scales corresponding to small assemblies of particles within a bulk. Under applied load, neighboring particles can experience vastly different forces, to the point where some particles may not see any load at all. Arguably, no other form of matter naturally shows this level of inhomogeneity. The mechanisms that generate this inhomogeneity revolve around the formation of localized networks known as force chains. These structures consist of linked arrays of particles that carry relatively large loads. They typically form a network or skeleton that percolates throughout the entire assembly, rapidly adapting and changing in response to deformation. They are thought to be an inherent feature of granular matter and form an important cornerstone in our path to understanding these materials. For example, even in the simplest case of Hertzian contact between spherical particles; the stiffness of any given contact is intimately related to the load that is carried [9]. From this perspective, the existence of force chains injects a significant level of complexity into the process of predicting bulk stiffness from real particle properties; as is currently done for the simplified case of constant linear particle

*Christopher.Wensrich@newcastle.edu.au stiffness (e.g., see Refs. [10-12]). The collapse and buckling of force chains also plays an important role in the kinematics of shear bands and the internal failure of granular assemblies in general [13-16].

Direct evidence of force chains in two dimensions can be found in numerous experiments based on the photoelastic effect (e.g., see Refs. [17-22]). In addition to the many spectacular images that have been produced with this method, significant quantitative understanding has been generated through the calculation of contact forces from birefringence patterns $[17,19,21]$. The inhomogeneity of contact forces at boundary surfaces has also been demonstrated by prior work using carbon paper [20,23] as well as dedicated instrumentation $[17,24]$. Three-dimensional observations have proved much more elusive. Some attempts have been made using three-dimensional photoelasticity with pore fluids of matching refractive index $[19,20]$; however, at present this work is still qualitative in nature. Some direct three-dimensional measurements at light loads have been made in colloidal systems using confocal microscopy [25,26].

Force chains result in distinctive distributions of contact force that have been the focus of significant interest in the past. General features that have been reported include an exponential decay of probability density for high contact forces and power-law relationship for small contact forces $[27,28]$. Many studies have also reported nonzero probability density for vanishing contact force [29]; however, this may be only a feature in systems with relatively low overall load. At higher loads, particle deformation may tend to distribute contact forces more evenly [17]. A variety of analytical approaches have been developed to describe the general features of the distribution as an outcome of various physical mechanisms (e.g., see Refs. [20,29-32] and others).

In this paper we present the first direct quantification of three-dimensional force chains in a physical granular system using a careful combination of neutron tomographic imaging and diffraction-based strain measurement. Through this process, we demonstrate that it is possible to uncover detailed information on the conditions experienced by individual particles within three-dimensional assemblies. 


\section{STRESS AND STRAIN MEASUREMENT USING NEUTRONS}

Neutrons interact directly with the nucleus of atoms as waves, with each atom in a crystal structure becoming a source for further radiation via Huygens's principle. Through the Laue equations, which are simplified as Bragg's law; $\lambda=2 d \sin \theta$, the angles of diffracted peaks formed in this way directly provide information on various interatomic spacings between lattice planes (see Fig. 1) and provide a window into the crystal structure within solid materials. Neutron diffraction is now routinely used to study both single crystals and polycrystalline materials. In the latter case, Braggs Law is satisfied only for those crystallites that (randomly) align with the measurement geometry. Polycrystalline neutron scattering is known as powder diffraction and is a very active area in crystallography [4].

Modern instrumentation (both time-of-flight and constant wavelength) is able to measure interatomic spacings with
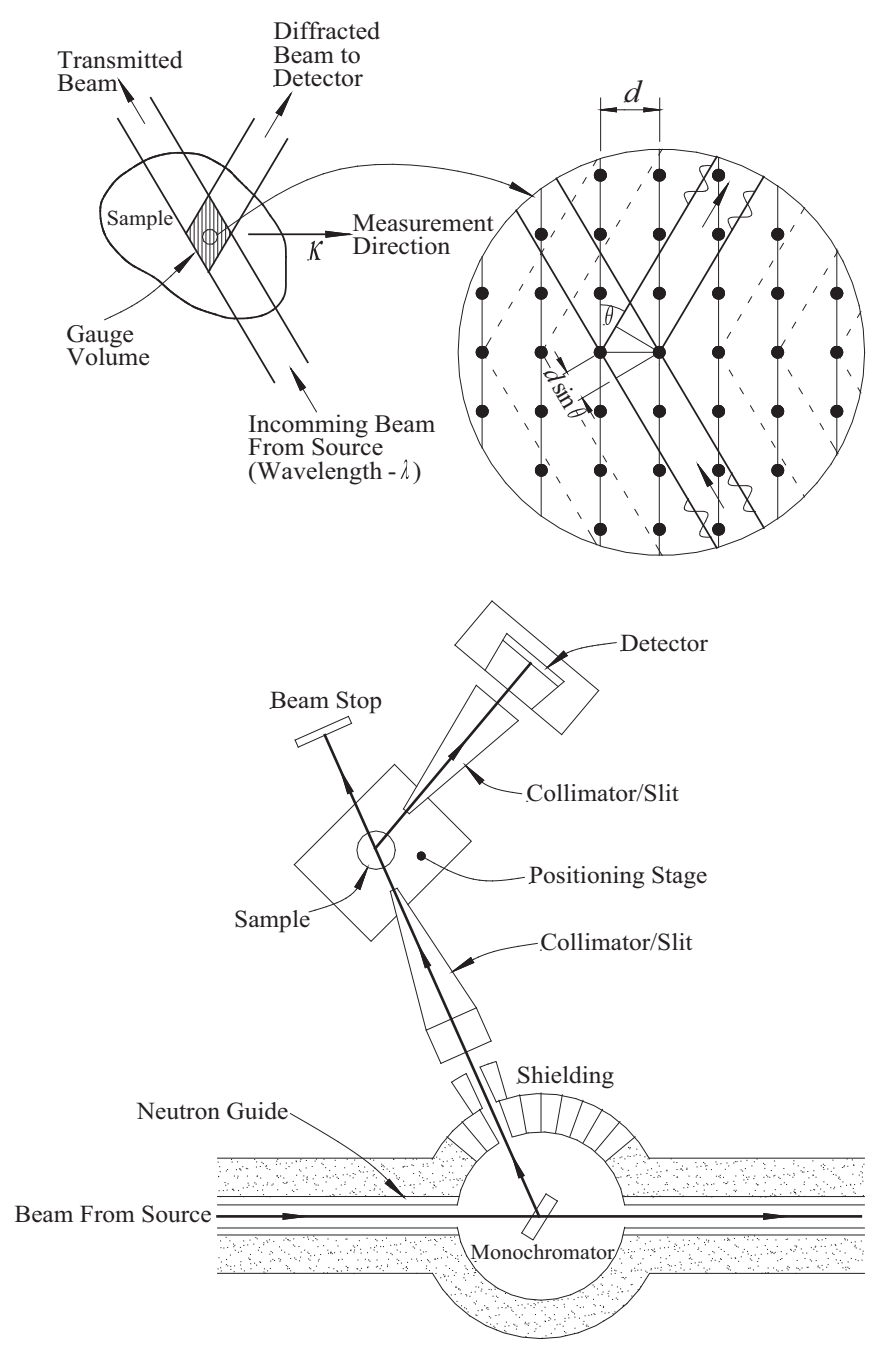

FIG. 1. Neutron diffraction occurs due to constructive interference related to the crystal structure of materials in directions defined by Braggs law. In a constant wavelength strain measurement, the angle of diffracted peaks is accurately measured to determine the interatomic spacing between crystal planes. sufficient accuracy to detect and measure lattice distortion due to elastic strain. Diffraction based strain measurement has become a valuable tool in both engineering and residual stress analysis as well as the fundamental study of the mechanics of solids.

Figure 1 illustrates a typical constant wavelength instrument for measuring strain using neutron diffraction. In this type of measurement, a monochromatic beam of neutrons is focused upon a sample and an area detector is used to measure the position of a single peak formed from a given set of crystal planes. A careful measurement of an initial spacing can be made in the absence of any load $\left(d_{0}\right)$, and strain is then measured as a relative change in this spacing:

$$
\epsilon_{\kappa}=\frac{d-d_{0}}{d_{0}} .
$$

Three features of such a measurement require special mention:

(1) $d$ and $d_{0}$ refer to the lattice spacing between planes that randomly align with the measurement geometry. More specifically, the spacing refers to the separation of planes with a normal direction corresponding to the bisection of the angle between the incoming and diffracted radiation (known as the scattering vector $\bar{\kappa}$ ). In other words, the measured strain represents the normal component of strain in a specific direction governed by the sample and instrument geometry.

(2) The measured strain is highly localized. It is specific only to a well-defined volume of material in the intersection of the paths between the neutron source and detector known as the gauge volume. The size of the gauge volume is easily controlled by the geometry of shielding slits (or collimators), which define the incoming and diffracted beams.

(3) Neutrons interact very weakly with many engineering materials. This allows them to penetrate significant distances and map out strain deep within samples.

Together, these three features provide for a powerful experimental technique. Through the measurement of sufficient strain components in a variety of directions and the spatial scanning of the gauge volume, it is possible to map out the distribution of fully resolved three-dimensional triaxial strain deep within solid materials.

It is important to note that the measurement refers only to the elastic component of strain within the material; plasticity within crystals does not affect the spacing. Consequently, Hookes Law applies to the calculation of stress corresponding to a strain measurement made in this way, regardless of the amount of plasticity experienced. This may seem deceptively simple; however, the complex mechanics of load sharing due to single-crystal elastic anisotropy can play a part here [22]. Each measurement is made on a small subset of crystals with planes that align with the scattering vector. Crystal grains orientated in this way may not necessarily have (on average) the same stiffness as their neighbors and correspondingly may see different strain to the bulk average. These mechanisms can significantly complicate the reconstruction of stress, although there are various approaches to deal with such problems $[3,4,6,22]$. With correctly chosen crystal planes, it is possible to make measurements that are unaffected by elastic anisotropy, and in this case stress $(\sigma)$ and strain $(\epsilon)$ are related 
by the isotropic version of Hooke's law,

$$
\sigma_{i j}=\lambda \epsilon_{k k} \delta_{i j}+2 \mu \epsilon_{i j},
$$

where $\lambda$ and $\mu$ are the usual Lamé elastic constants.

In summary, through this technique it is possible to directly determine the detailed distribution of the full triaxial stress tensor within an in situ-loaded solid sample. Measurements made in this way are unambiguous, irrespective of the many variables associated with the system (e.g., material properties, load case, geometry, etc.). In other words, when carefully conducted, this technique is purely experimental and independent of models apart from elasticity theory. No calibration is required.

\section{STRESS MEASUREMENT IN GRANULAR ASSEMBLIES}

In some sense, the application of neutron diffraction techniques to measuring stress in crystalline granular materials is unremarkable; neutrons are diffracted by the solid material within the particles as they would be by the same material in continuous form. Average (or bulk) stress distributions in various powders have been directly measured using this technique by employing gauge volumes much larger than the typical particle size [7,8] [Fig. 2(a)]. In this way, the measurement provides the average elastic strain and the corresponding bulk stress (once adjustments for the volume solid ratio are made) over all particles within the gauge volume. The gauge volume becomes a representative volume element of the granular continuum similar to that used in averaging or homogenization techniques applied to discrete models (e.g., see Refs. [33-35]). The only significant difference that exists between a regular solid and a granular assembly is in the load sharing mechanisms surrounding the effects of single-crystal elastic anisotropy. These mechanisms are much less understood in granular assemblies.

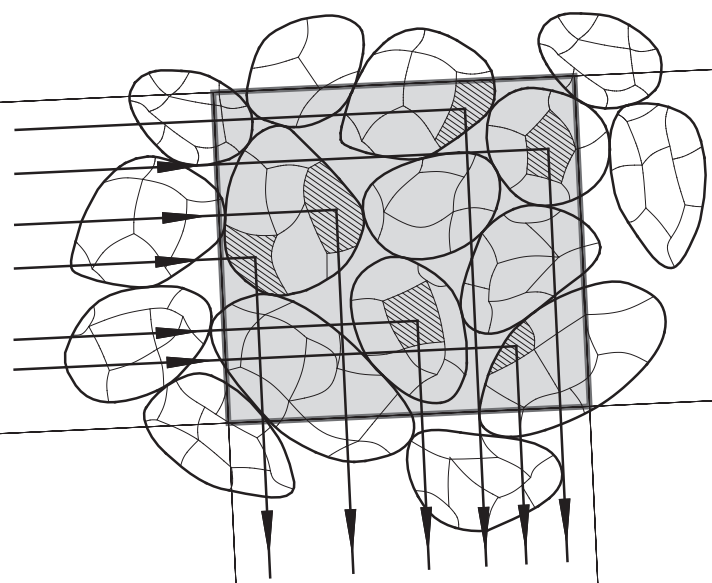

In this paper we turn our attention from bulk measurements to applying the technique to granular assemblies in discrete form. Our aim here is not to map out the distribution of bulk stress within an assembly but rather to examine the stresses experienced at the scale of individual particles. Simplistically, this can be achieved by employing a gauge volume that corresponds to the particle size; however, the level of inhomogeneity at this length scale presents some interesting challenges.

\section{A. Preliminary work}

As a preliminary investigation, a set of strain measurements were carried out in a monodisperse assembly of $3.16 \mathrm{~mm}$ (1/8 inch) steel ball bearings compressed within a cylindrical aluminium die $(\varnothing 26 \times 70 \mathrm{~mm})$. These measurements were carried on the KOWARI strain diffractometer at the Bragg Institute within the Australian Nuclear Science and Technology Organisation (ANSTO) [36] using the 211 reflection in $\alpha$-ferrite and a neutron wavelength of $1.66 \AA$ (i.e., $90^{\circ}$ geometry). Load was applied to the assembly via an Instron load frame mounted to the sample stage of the instrument to a bulk stress of $180 \mathrm{MPa}(100 \mathrm{kN})$.

The ball bearings were purchased in a hardened state before being annealed in an inert argon atmosphere at $560^{\circ} \mathrm{C}$ for $1 \mathrm{~h}$ and allowed to cool at a fixed rate of $10^{\circ} \mathrm{C} / \mathrm{h}$. This process was aimed at yielding a uniform microstructure within the balls and the tempering of martensite to alleviate peak broadening due to the internal stresses and grain size effects associated with this phase. Through this process, narrower diffraction peaks more favorable for strain measurement are obtained.

Using a gauge volume of $3 \times 3 \times 3 \mathrm{~mm}^{3}$, axial strain was measured over a regular three-dimensional grid in a $17.5 \times 17.5 \times 36 \mathrm{~mm}^{3}$ rectangular prism wholly contained within the assembly. The spacing between sample points was 3 and $2.5 \mathrm{~mm}$ in the axial and lateral direction, respectively.

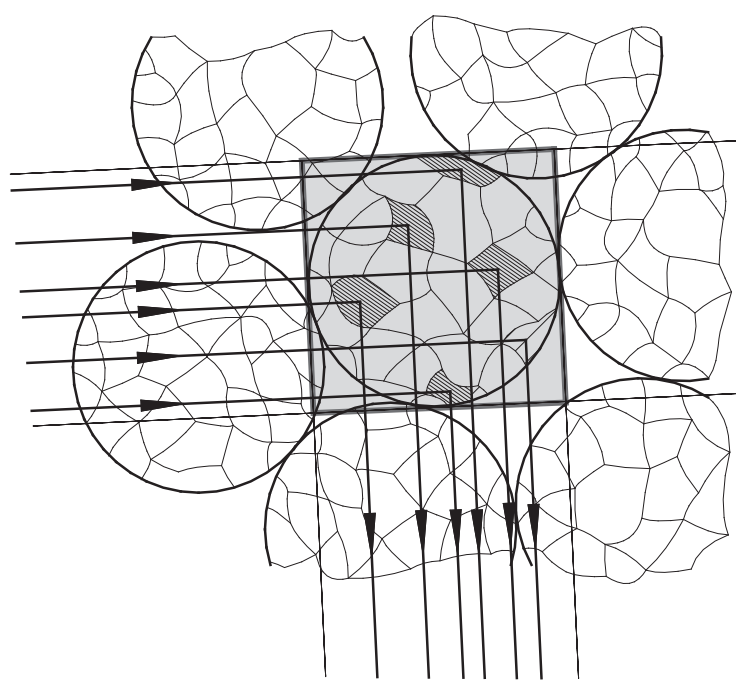

FIG. 2. All crystal grains within the gauge volume that randomly align with the geometry of the instrument take part in the measurement. (a) For bulk measurements a gauge volume much larger than the particle size can be employed to average across a larger assembly of particles. Note that for illustrative purposes the particle size shown is comparable in magnitude to the gauge volume; in reality, there is usually several orders of magnitude between the gauge volume and particle size in a bulk measurement. (b) In the present work we are concerned with the stress within individual particles; to study the material at this scale we employ a gauge volume that corresponds closely to the particle size. 
The resulting axial strains, normalized for the exponential decay of stress along the bed, are shown in Fig. 3. The normalization refers to the ratio of observed strain at any point to the mean axial strain in that layer of the threedimensional scan. It was immediately apparent that a high level of inhomogeneity exists within the data. The observed strains ranged from negligible amounts to a maximum of $1300 \times 10^{-6}$ (i.e., $1300 \mu \epsilon$ ). It is also apparent from Fig. 3 that the inhomogeneity contains some structure, indicating the existence of force chains.

The results of this preliminary investigation confirmed the presence of force chains in this system and were encouraging from the point of view of using neutron diffraction for uncovering discrete information within granular assemblies. However, two significant issues with the measurement were noted.

First of all, the regular grid over which the measurements were made bears no relationship to the location of individual particles. At any random location, the gauge volume is likely to contain random portions of several particles that experience very different strain. We have no way of knowing how such an average should relate to the conditions experienced by individuals. Second, we would expect that in any given measurement a random and significant portion of the gauge volume would contain voids. This situation can introduce significant uncertainty into the strain measurement through a mechanism known as "partial illumination" as explained in detail later in this paper. To avoid these issues, it was apparent that a more sophisticated approach was required.

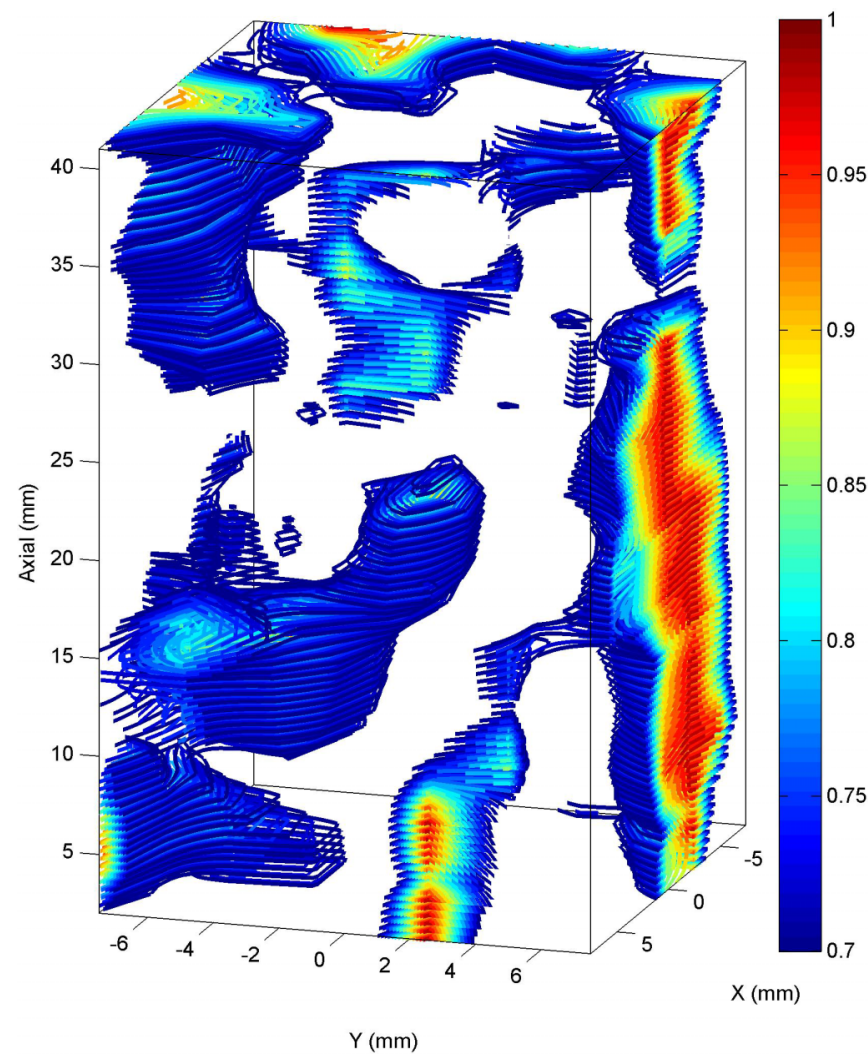

FIG. 3. (Color online) Normalized axial strain measurements within a cylindrical monodisperse assembly of steel ball bearings under load. Contours have been interpolated from experimental measurements located over a regular three-dimensional grid.

\section{B. A more refined approach}

The problems associated with the preliminary measurement can be largely avoided if the gauge volume can be accurately centered on individual particles as shown in Fig. 2(b). In this way, each measurement would refer to the average stress or strain within an individual particle with only a small influence of any neighbors that happen to overlap the gauge volume. Note that the strain field within a single particle is highly inhomogeneous. Significantly higher stress is experienced at the points of contact compared to the average. This inhomogeneity within particles has no direct impact on the measurement; neutrons uniformly sample all solid material within the gauge volume. The corresponding calculated stress represents a volume average over the included material. A detailed discussion on this point can be found in Ref. [6].

The development of a systematic approach to positioning the gauge volume in this way would allow the exhaustive mapping-out of average stress within every particle in an assembly.

Our approach to achieve this was as follows:

(1) Form an assembly of monodisperse spherical particles within a die and apply a prescribed load.

(2) With the load applied, obtain three-dimensional neutron tomographic images of the assembly.

(3) Using appropriate image processing techniques, determine the position of each particle in three-dimensional space.

(4) Using a strain diffractometer with a gauge volume corresponding to the size of an individual particle, drive to the coordinates of each particle and measure sufficient components of strain to determine its average elastic strain tensor.

(5) Using Hookes law, individually calculate the corresponding average stress tensor for each particle.

At least at this stage, it would be unrealistic to attempt to apply this approach to a typical granular system consisting of millions of polydisperse irregularly shaped particles. Instead, much as is done in current modeling techniques, we will examine a simplified system consisting of a modest assembly of spherical particles.

\section{EXPERIMENTAL SYSTEM}

The particle system at the focus of this work consists of an assembly of 549 individual $3.16-\mathrm{mm}$ (1/8 inch) precision steel ball bearings, prepared in an identical fashion to the those in the preliminary experiment in terms of heat treatment. These steel balls were poured into a 30 -mm-diameter selfloading cylindrical die, forming a bed around $22 \mathrm{~mm}$ deep (as shown in Fig. 4). Load was applied to the top surface of the loose poured assembly via a single-acting aluminium plunger, manually actuated via a hardened steel set-screw and nut $(\mathrm{M} 24 \times 1.5)$. The compressive force from the setscrew was passed through a large $(25-\mathrm{mm})$ hardened steel ball bearing in an effort to avoid torque transmission to the plunger. Excluding the setscrew, ball bearing, and nut, all parts of the die were constructed from a high-strength aluminium alloy (7075-T6), chosen for its relative transparency to thermal neutrons.

The total load on the particle bed could be accurately measured at any time using a set of three strain gauges affixed to a turned-down section of the die head. Using a system of 


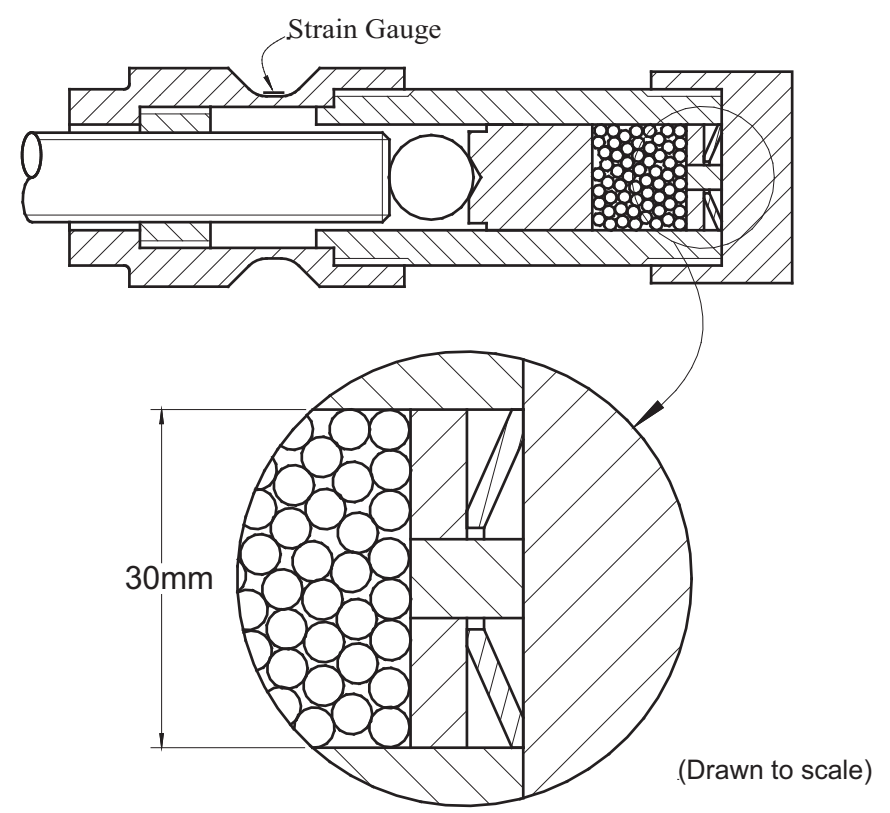

FIG. 4. The particle bed was contained within a 30-mm aluminium self-loading die. $60 \mathrm{kN}$ of load was applied via a high-strength steel setscrew acting on a hardened steel ball bearing. At the base of the die (shown inset), a "hard post" was provided by the inclusion of an aluminium Belleville washer.

large spanners and extension bars, $60 \mathrm{kN}$ of force was applied to the bed; this load remained constant throughout the entire experiment (and remains applied to the assembly to this day). The key feature of this self-loading system is that once the prescribed load is applied, the die becomes self-contained and compact. This provides excellent mobility within the diffractometer, allowing rapid and automated repositioning via the sample stages of the instrument and ensures identical sample conditions on different instruments.

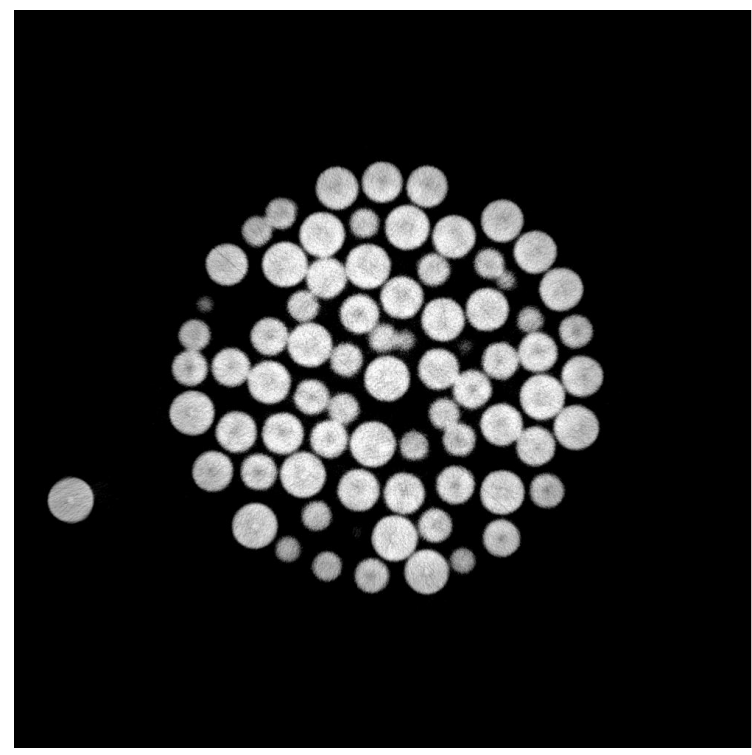

At the base of the die, a number of small components were designed and installed in an effort to focus the generation of force chains toward the center of the die (as shown in the inset in Fig. 4). This system consisted of three separate parts: a solid cylindrical aluminium post in the center of the die $(\varnothing 7 \times 10 \mathrm{~mm})$, a 5 -mm-thick concentric aluminium outer ring with clearance (sliding) fits between the central post and the outer die wall, and a single aluminium Belleville washer that supports the outer ring. As load is applied to the outer ring, the Belleville washer deforms and the central post projects into the particle assembly. As has been observed and studied previously [21,37], the hard post at the center of the die base preferentially loads particles in contact with it and initiates force chains in the material above. It is important to note that force chains would most likely exist without these provisions (they are inherent in granular matter); however, through this mechanism they can be focused to a known point.

In addition to the balls inside the die, four $3.16-\mathrm{mm}$ steel balls were embedded into the external surfaces of the die at known positions. These additional balls served as fiducial markers, providing accurate position and orientation information for sample alignment.

\section{CT RESULTS AND IMAGE PROCESSING}

The newly commissioned neutron-imaging instrument known as "DINGO" within the Bragg Institute at ANSTO [38] was used to obtain a three-dimensional tomographic scan of the particle bed and die system. High contrast tomographic slices of the bed were obtained with a resolution of $50 \mu \mathrm{m}$ in each coordinate direction. The significant difference in attenuation levels between the aluminium die and steel balls provided for simple thresholding of the images to obtain information on the isolated bed and fiducial markers. An example image from this process after thresholding is shown in Fig. 5(a).

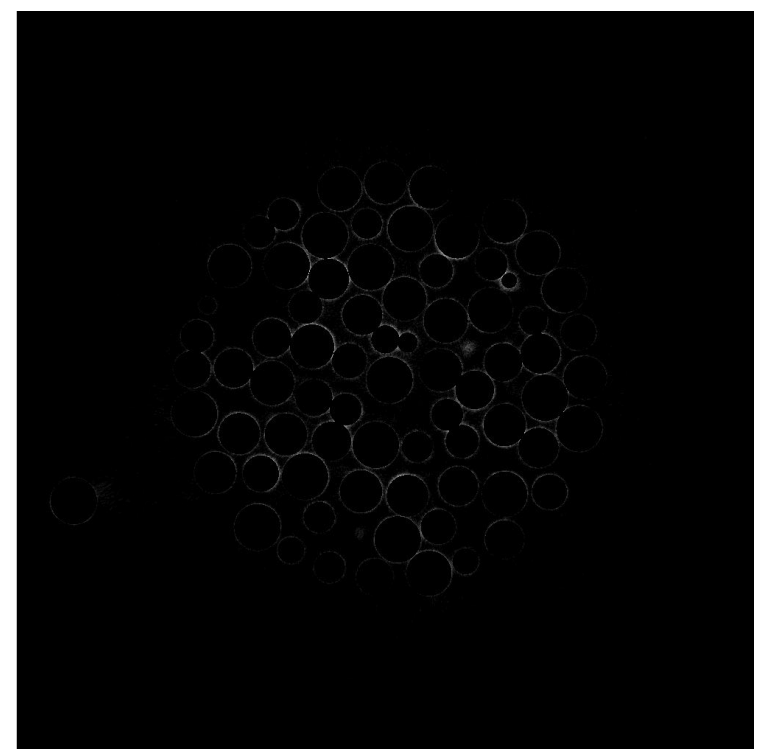

FIG. 5. (a) An example of a tomographic slice from the neutron imaging on "DINGO." The particles within the die can clearly be seen as well as one of the fiducial markers embedded in the die wall. (b) The remainder of the image after the balls located by the optimization algorithm (in 3D) are blackened-out. 
Each individual ball within the bed was then located in three dimensions within the image stack using a computational approach previously developed for analyzing x-ray CT images. In summary, particles are sequentially located within the bed by maximizing an objective function formed by the sum of voxels contained within a sphere representing the particle. After each particle is fitted to the scan, the voxels within are set to zero to penalize future overlap. Full details of the algorithm are outlined by Wensrich [39].

After fitting each particle, the remainder of the tomographic images depict the blurry penumbra formed by the imaging process as observed in Fig. 5(b). The accuracy of fitted positions can be gauged qualitatively by these shadows, or quantitatively by the maximum overlap between any two particles within the bed. Typically, the algorithm is able to locate particle with an accuracy that is similar to, or slightly better than, the resolution of the scan.

The imaging process and subsequent processing was able to accurately locate all 549 particles within the bed as well as the four fiducial markers. Out of the thousands of contacts within the bed, the maximum overlap between any two balls was $140 \mu \mathrm{m}$. This number represents approximately twice the absolute worst case position error. Given that several thousand contacts exist in the assembly, we can estimate that the expected error would be a fraction of this value. General statistics of the final packing include a volume solid ratio of $58.6 \%$ and an average coordination number of $7.0 \pm 0.1$. It should be noted that the assembly is contained within a volume that is only 10 times the particle diameter. It is likely that there are significant wall effects that have an impact on the structure and amount of porosity.

\section{STRAIN MEASUREMENT}

\section{A. Sample alignment}

The loaded die was then mounted on the positioning table of the KOWARI strain diffractometer. The sample was held in a 6-jaw chuck mounted to a separate rotation stage allowing rotation about the $y$ axis as shown in Fig. 6. The angle of rotation about this axis is denoted $\Omega_{e}$ in the following.
The results of the neutron imaging provided the threedimensional coordinates of each ball in the natural Cartesian coordinate system associated with the stack of images. Accurate positioning of the particles within the gauge volume of KOWARI required the development of an appropriate coordinate transformation between the image coordinate system and the coordinate system defined by the sample stage motor drives. To achieve this we relied upon the positions of the four fiducial markers and the process outlined in the Appendix at the end of this paper.

The result of this process provided a single threedimensional coordinate transformation written in terms of the angular position of the rotation stage $\Omega_{e}$. Spot checks on the fiducial markers in a number of angular positions provided confidence that all particles in the assembly could be positioned within the gauge volume to an accuracy well within $100 \mu \mathrm{m}$ in any orientation.

\section{B. Strain measurement and stress reconstruction}

A series of strain measurements were made using the 211 reflection in $\alpha$-ferrite with a gauge volume of $3.2 \times 3.2 \times 3.8 \mathrm{~mm}^{3}$ (measured at the sample) and a neutron wavelength of $1.66 \AA$. This configuration provides for a $90^{\circ}$ geometry between the incoming and diffracted beams, i.e., an approximately cubic gauge volume.

The measurement of a single-strain component associated with a given particle relies upon fitting a Gaussian to a measured histogram. The uncertainty associated with this measurement is directly linked to the shape of the peak and the neutron count. In all of this work a minimum neutron count was set to achieve a strain uncertainty below $5 \times 10^{-5}$. As a result of attenuation, the period of time necessary for this to be achieved varied depending on the position of the particle in the assembly; however, a typical sample time was of the order of several minutes.

The determination of the full strain tensor relies upon the measurement of a sufficient number of independent components of normal strain. To simplify this process, strain measurements were made within the $x-y$ plane at $45^{\circ}$ to the $x$ and $y$ directions. In this setup, different components of strain
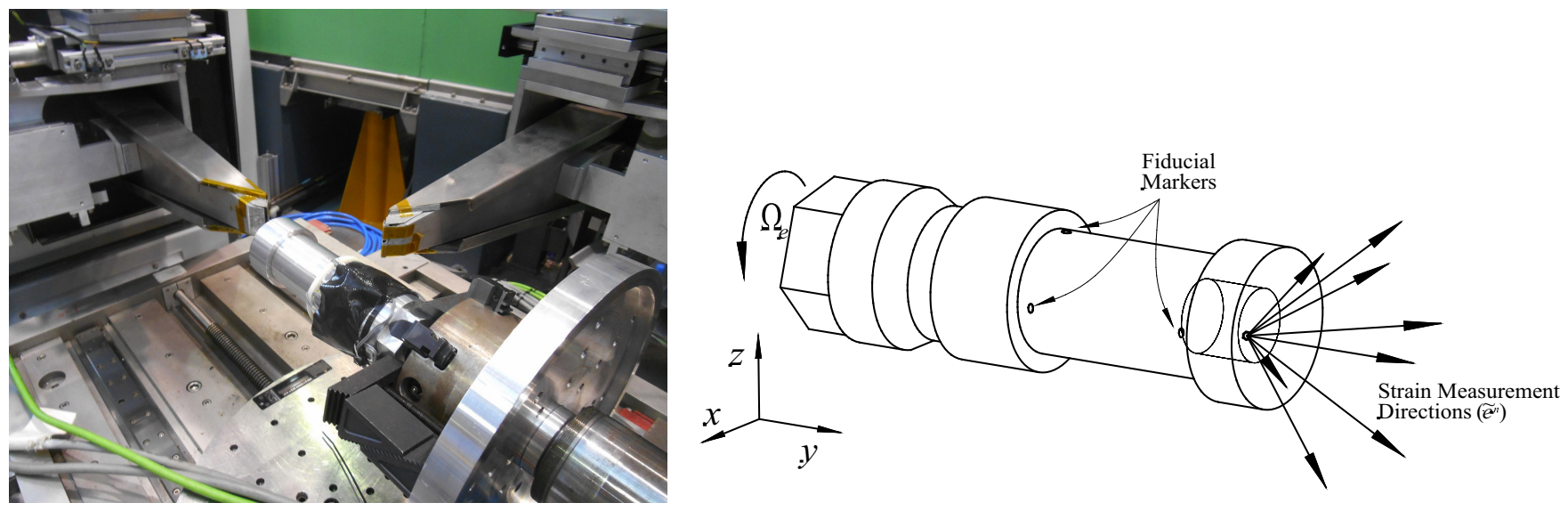

FIG. 6. (Color online) The sample was oriented on the KOWARI sample stage with its central axis aligned to the $y$ axis. In this orientation various components of strain can be measured by rotating the sample about this axis. Sample alignment was achieved using the fiducial markers embedded in the die walls. 
can be measured by simply varying the position of the rotation stage. Using this approach, strain measurements for each of the 549 particles were made at six angular positions; $\Omega_{e}=0,60$, $120,180,240$, and $300^{\circ}$. In addition to these six components, a measurement of strain in the axial direction, as well as a single component at $\Omega_{e}=90^{\circ}$ at $30^{\circ}$ from the $y$ axis were also made. The directions associated with the eight measured strain components are illustrated in Fig. 6(b).

We can then calculate the six unknown components of the strain tensor $\epsilon_{i j}^{p}$ for each particle $p$ as the least-squares best fit to the eight measured components of normal strain $\epsilon^{p n}$. Designating the directions of the measured components as $\hat{e}^{n}$ for $n=1,2, \ldots, 8$, the sum of squares error associated with particle $p$ can be written

$$
\Lambda=\sum_{n=1}^{8}\left(\epsilon^{p n}-\epsilon_{i j}^{p} e_{j}^{n} e_{i}^{n}\right)^{2} .
$$

This error is to be minimized by an appropriate choice of $\epsilon_{i j}^{p}$, which occurs when $\frac{\partial \Lambda}{\partial \epsilon_{i j}}=0, \forall i, j$, or

$$
\sum_{n=1}^{8} e_{j}^{n} e_{i}^{n}\left(\epsilon^{p n}-\epsilon_{k l}^{p} e_{k}^{n} e_{l}^{n}\right)=0, \quad \forall i, j .
$$

The solution of the resulting set of six simultaneous equations (one for each unique pairing of $i$ and $j$ ) provides the strain tensor for particle $p$. By repeating this process, the strain tensor within every particle in the assembly was determined. The mean stress within each particle was then calculated using Eq. (1) with $\lambda=102 \mathrm{GPa}$ and $\mu=80 \mathrm{GPa}$. Note that the normal compliance of the (211) lattice planes are very close to the isotropic mean.

The calculated stress in each particle depends directly on the value of $d_{0}$ used in the analysis. In an effort to obtain the most consistent result, a value of $d_{0}$ was chosen to provide an average axial stress in the top layer of particles that corresponds to the applied load. This calculation was performed on only those particles in direct contact with the plunger, for which the average stress can be estimated from the mean stress theorem as [33]

$$
\left\langle\sigma_{22}\right\rangle=\frac{F}{\frac{4}{3} \pi r^{3} N}\left(\left\langle y^{c+}\right\rangle-y^{c-}\right),
$$

where $F$ is the applied load acting at $y^{c-}$ on the top layer of $N$ particles, and $\left\langle y^{c+}\right\rangle$ is the average $y$ coordinate of all other contacts on these balls.

\section{FORCE CHAINS IN 3D}

A dominant feature of the measured stresses is the widespread inhomogeneity in the direction and magnitude of the stresses. This observation was in-line with expectations drawn from the prior two-dimensional studies of the force chain phenomenon referenced earlier. With an average stress so high it is interesting to note that bulk plasticity in the form of particle motion and reorientation did not allow sufficient stress redistribution to destroy the force chains.

The effects of the hard post were clear to see in the results. Being that the results consist of 549 individual threedimensional stress tensors, they are quite difficult to present
Axial Stress
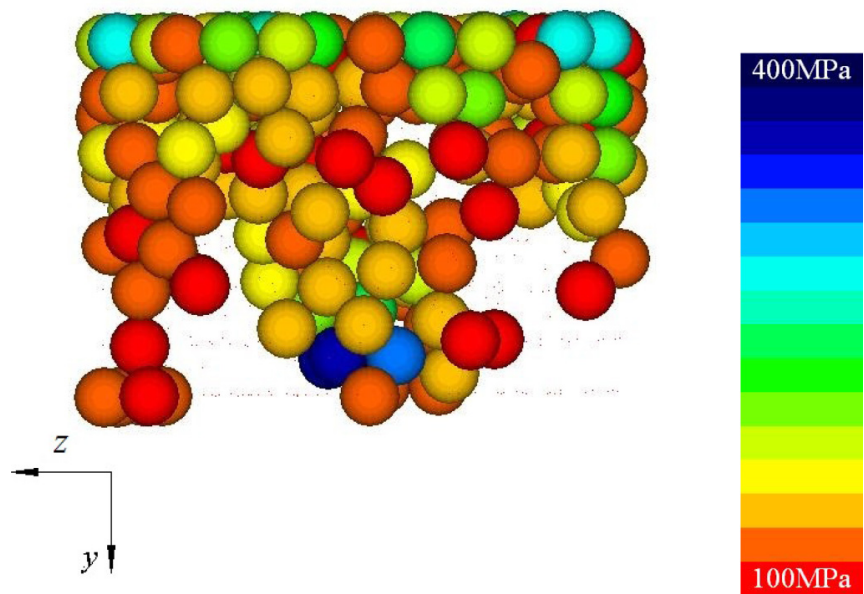

FIG. 7. (Color online) Axial stress within the assembly of particles. Here the bed is shown with its axis vertical with the plunger acting on the top surface. Only those particles with more than $100 \mathrm{MPa}$ of compressive stress are shown. The positions of others are indicated as points. The existence of a number of particle chains with relatively high stress can clearly be seen. The most significant of these chains originates at the hard post at the center of the bottom of the assembly.

fully in journal format. A convenient summary is given in Fig. 7, which shows the distribution of axial stress within the bed for all particles that experience more than $100 \mathrm{MPa}$. For additional clarity, the same data are replotted in Fig. 8 as six $x-z$ plane sections at different axial positions. The axial component shown here represents the most accurate component measured (see Sec. VIII) and corresponds to the direction of loading. In each of these figures, highly loaded particles in contact with the hard post can clearly be seen at the base of the assembly (e.g., the $y / d=6$ section in Fig. 8). In Fig. 7 we see that these particles have been pushed into the bed slightly as the assembly deformed under load. In terms of both hydrostatic and deviatoric components, these particles were the most highly loaded in the entire assembly. The average axial component of stress in the most highly loaded ball was in excess of $400 \mathrm{MPa}$. At such high stress levels we would expect that some plastic deformation at points of contact would be apparent, at least in the aluminum post. As discussed earlier, and elsewhere [6], this form of plasticity has no overall effect on the measurement of stress using this method.

The high stress in the particles in contact with the post was observed to be distributed into the bed above over a relatively short distance. A central column of highly loaded balls is observed to propagate upward from the post, through the bed to the top surface. This column represents a large force chain (or collection of force chains) that supports the majority of the load at the base. Outside of this central column, several other isolated force chains can also be seen, predominantly located along the walls of the die. At the threshold shown, one of these isolated chains spans the entire length of the bed-from the base to the face of the plunger.

High levels of axial stress were also observed in the particles in direct contact with the plunger. This is due to the fact that the 

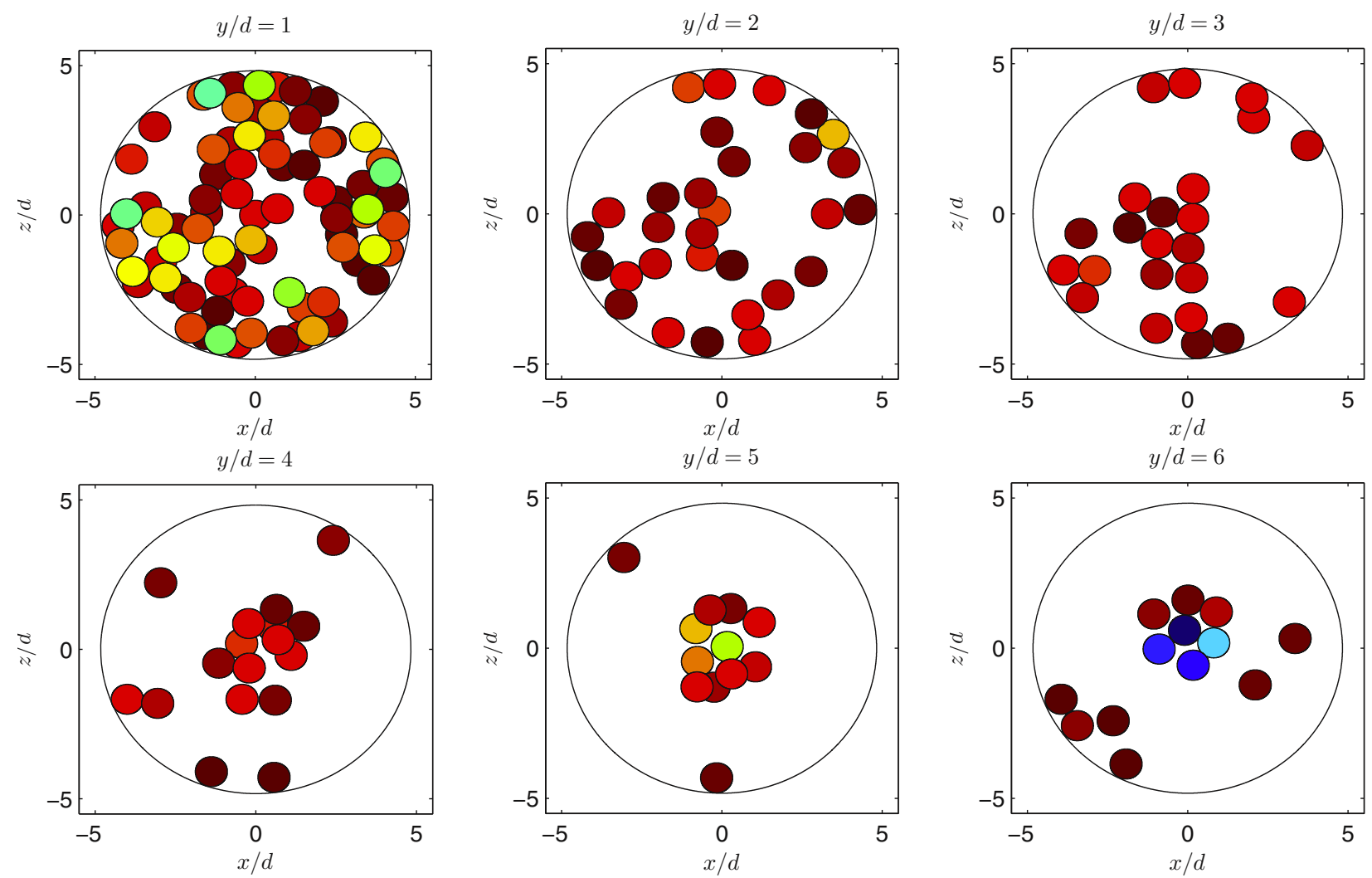

FIG. 8. (Color online) Axial stress within the assembly shown in terms of highly loaded balls in a number of successive layers within the bed. As described in the legend of Fig. 7, only those balls with more than $100 \mathrm{MPa}$ of compressive stress are shown. Colors refer to the same mapping to axial stress as in Fig. 7.

contacts that are formed here predominantly have their normal component in the axial direction; the hydrostatic component of stress in these particles is not significantly different from the next layer.

Shear stress in the axial-radial direction is shown in Fig. 9. High levels of shear stress are observed in the particles in direct contact with the walls of the die, while shear stress in the opposite direction is observed in the central column. This is assumed to be in response to the friction developed between the particles and die wall and the internal friction developed by the relative axial motion of the central column due to the hard post.

The observed probability distribution function for various components and measures of stress are shown in Fig. 10. All of the shear components were found to have Gaussian distributions. In contrast, normal components and measures of overall load (e.g., hydrostatic and von Mises stress) showed long decaying tails into high stress levels - a clear confirmation of the inhomogeneity associated with force chains. Log-normal distributions were found to fit the observed behavior of these components reasonably well within statistical scatter. The fitted distributions are shown alongside the data in Fig. 10. In each case, the only significant outliers were the four particles in direct contact with the hard post. Parameters associated with these curve fits are given in Table I.

This finding is in some contrast to the exponential decay at high loads that has previously been reported by Radjai et al. [27], Liu et al. [20], and Majmudar et al. [18] (among others). However, it should be noted that the results reported here are average particle stress rather than contact force; although one might expect that the trend should be similar. Indeed, the similarity between the shape of force and pressure

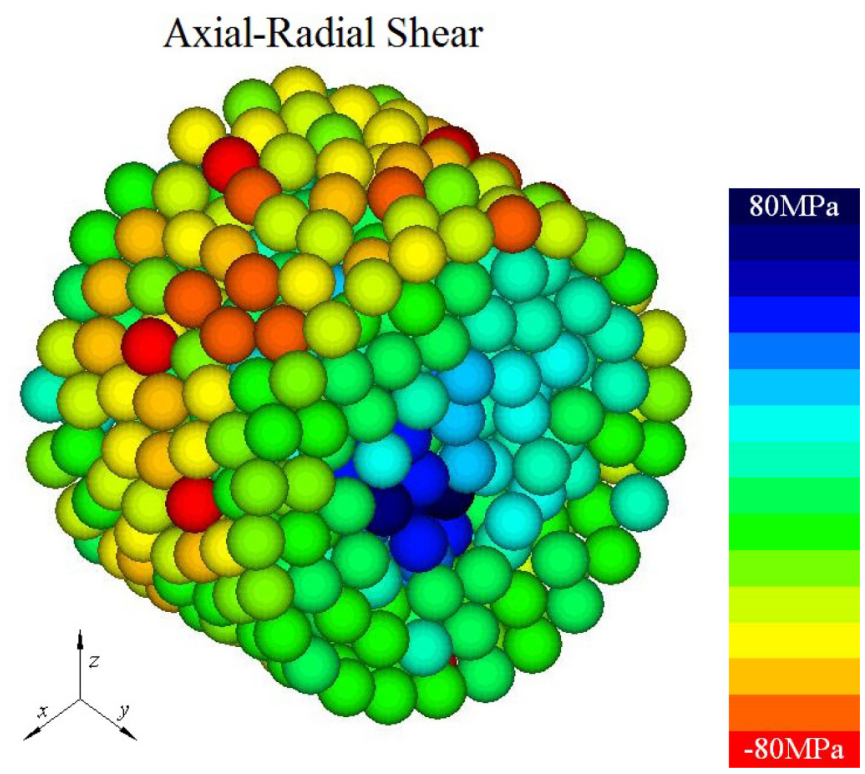

FIG. 9. (Color online) Shear stress in the axial-radial direction for all particles in the assembly. High levels of shear stress are observed in the particles in contact with the walls and in the opposite direction in the central force chain at the center of the die. 

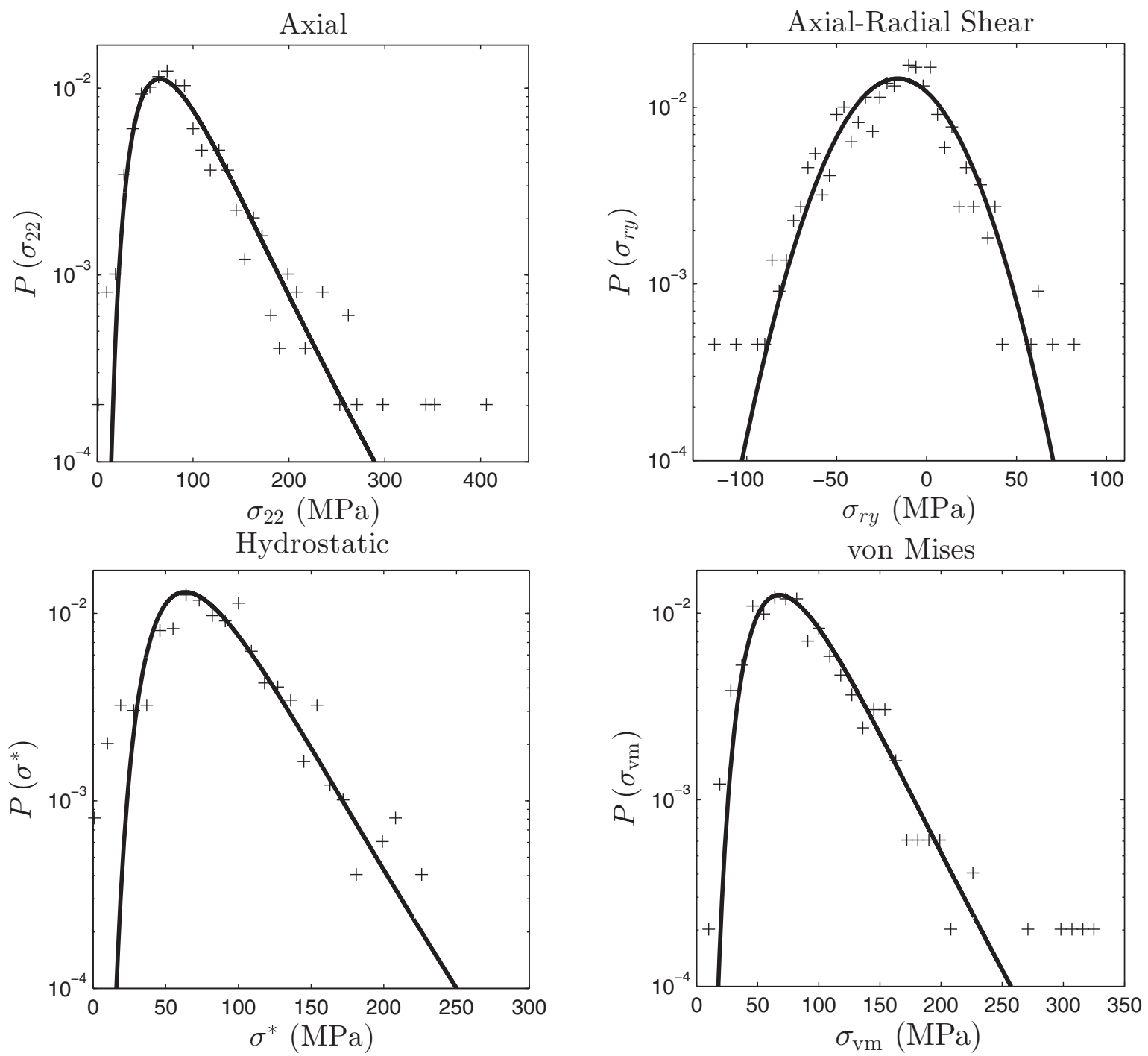

FIG. 10. Probability distribution functions for axial $\left(\sigma_{22}\right)$, shear $\left(\sigma_{r y}\right)$, hydrostatic $\left(\sigma^{*}=\sigma_{i i} / 3\right)$, and von Mises effective stress $\left(\sigma_{v m}\right)$ within the assembly. Also shown are fitted normal and log-normal distribution functions; parameters are provided in Table I.

distributions has been observed before by Tighe et al. [40], who demonstrated through entropy that Gaussian-like tails can be expected in both cases; at least in the case of two-dimensional ordered structures.

Unfortunately, due to the orientation of the measured strain components, the uncertainty in the lateral components of stress currently prohibits the accurate calculation of the threedimensional force network within the assembly. If additional lateral components of strain can be measured in the future, it is expected that such a calculation will be possible using the approach outlined by Andrade and Avila [41] and Hurley et al. [42]. It is also apparent that a probability density of zero at vanishing normal stress is observed in our results. As explained by Howell et al. [17], this may be due to the fact that our assembly is under a relatively large amount of external load.

\section{POTENTIAL SOURCE OF ERRORS}

In addition to the usual errors associated with counting statistics and peak-fitting algorithms, strain measurements in granular materials suffer from a number of inherent errors

TABLE I. Fitted probability distribution function parameters (stress in [MPa]).

\begin{tabular}{lcccc}
\hline \hline \multicolumn{2}{c}{ Log-normal: $P(\sigma)=\frac{1}{\sigma a \sqrt{2 \pi}} e^{-\frac{1}{2 a^{2} \ln ^{2}\left(\frac{\sigma}{b}\right)}}$} & & Normal: $P(\sigma)=\frac{1}{c \sqrt{2 \pi}} e^{-\frac{1}{2 c^{2}}(\sigma-d)^{2}}$ \\
Component & $a$ & $b[\mathrm{MPa}]$ & Component & $c[\mathrm{MPa}]$ \\
\hline Axial & 0.485 & 82.5 & Axial-radial shear & 27.4 \\
Hydrostatic & 0.440 & 82.3 & & -16.2 \\
von Mises & 0.430 & 81.4 & & \\
\hline \hline
\end{tabular}


associated with the porosity. With care, these errors can usually be eliminated (or at least minimized) when making bulk measurements. With a gauge volume much larger than the particle size, the random nature of the porosity means that the material appears homogeneous. In this work we employed a gauge volume that corresponds to the size of a single particle and at this scale the material is far from homogeneous. This can introduce additional errors in strain measurements as follows.

\section{A. Partial illumination}

The term partial illumination (or partial burial) usually refers to an apparent peak shift that occurs when the gauge volume overlaps an external boundary of a sample. The amount of peak shift to be expected by a given geometry is well characterized and can be accounted for in some standard cases (e.g., see Refs. [43-45]). In the case of a granular material, a very similar effect can occur on a random basis at any point within an assembly (not just on an external boundary).

In detail the effect is related to factors including the geometry of the instrument and slit system, the properties of the monochromator (e.g., mosaic spread), and the location of the "unfilled" portion within the gauge volume [43]. All of these details are important for a complete understanding of the phenomenon; however, a very simple (and instructive) explanation is also available; the peak shift is related to any deviation of the center of mass of the material within the gauge volume from the geometric center. In general (attenuation effects aside), the average neutron is diffracted from this center of mass. If, for any reason, the center of mass moves away from the geometric center, the geometry of the measurement changes and the location of the peak at the detector can move by a small amount. The relative amount of the shift is perceived as a pseudostrain, or error in the strain measurement.

Within our measurement the movement of the center of mass can occur in two ways:

(1) Any error in positioning can lead to a deviation between the center of the ball in question and the center of the gauge volume as shown in Fig. 11(a).

(2) The particles in the assembly are spherical, whereas the gauge volume can be approximated as a rectangular prism. In the general case of a single particle within a bed, a small portion of the neighboring particles may overlap the gauge volume [see Fig. 2(b)] and take part in the measurement. Their intrusion into the gauge volume has the effect of randomly disturbing the center of mass. Note that the strain within these small regions will also contribute to the final strain measurement; however, it is reasonable to expect that their contribution will not be too dissimilar to the ball in question (after all they share the same contact force).

\section{B. Attenuation effects}

A related problem is due to the effects of attenuation within a randomly packed bed of particles. To focus the gauge volume on a given particle within the bed it is necessary to pass the incoming and diffracted beams through a series of neighbors. At all points along these paths the neutron beam is being attenuated through the combined effects of absorption and scattering (both coherent and incoherent). With a gauge
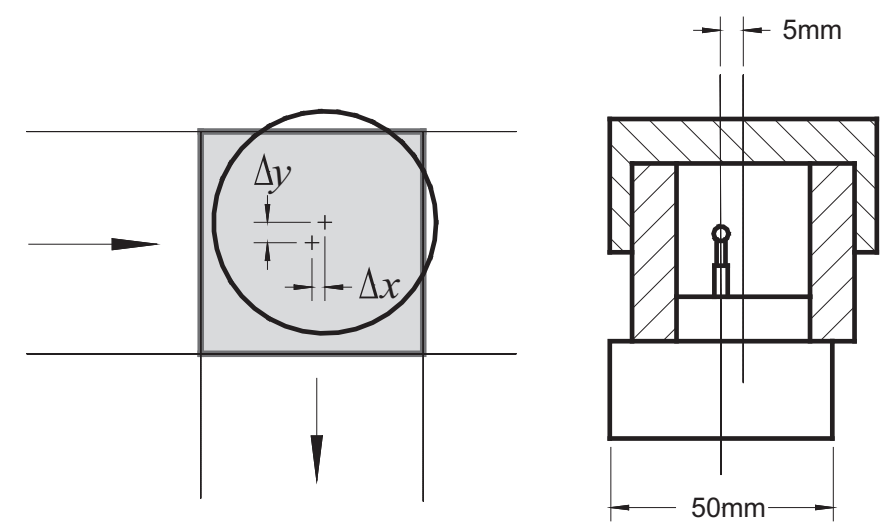

FIG. 11. As the size of the gauge volume approaches the particle size, additional errors can be expected due to partial illumination and attenuation effects. (a) Partial illumination effects are related to positional misalignment of the particle within the gauge volume. (b) A mock die constructed to investigate and quantify the magnitude of errors due to attenuation and the intrusion of neighboring particles into the gauge volume (drawn to scale).

volume equivalent in size to a single particle, the structure of these paths cannot be considered homogeneous. And so we should expect that the intensity at the gauge volume will reflect this inhomogeneity, resulting in some portions being slightly oversampled when compared to others. In essence this can be viewed as a similar effect to the partial illumination problem; nonuniform attenuation due to the particle bed may mean that the average neutron does not quite originate from the center of the gauge volume. Once again, this is perceived as a small random peak shift or pseudostrain.

\section{Error estimation}

In an effort to characterize the extent of the errors described above, a short experiment aimed at separating and quantifying each effect was undertaken. This experiment was focused on measuring a $d$ spacing from a single unloaded particle with and without a surrounding particle bed. The particles used in this process were identical to those in the main experimental system.

A mock die was manufactured with equivalent dimensions and materials to the experimental system [see Fig. 11(b)]. This mock die featured a thin internal post on which a single 3.16-mm steel ball was affixed using a cyanoacrylate adhesive. The post (and ball) was accurately aligned centrally to the die base to allow accurate alignment within the strain diffractometer, while the die wall was offset by $5 \mathrm{~mm}$ from this central axis. This arrangement allowed various incoming and outgoing path lengths to be examined without disturbing the alignment of the single ball within the diffractometer (rotation about the central axis does not affect the position of the ball).

This arrangement was mounted on the KOWARI sample stage with an equivalent instrument setup to the strain measurements. The $d$ spacing corresponding to the 211 reflection was then measured for the single ball on the post under the following conditions:

(1) In the absence of neighbors at six equally spaced angular positions over one full axial revolution of the die. 

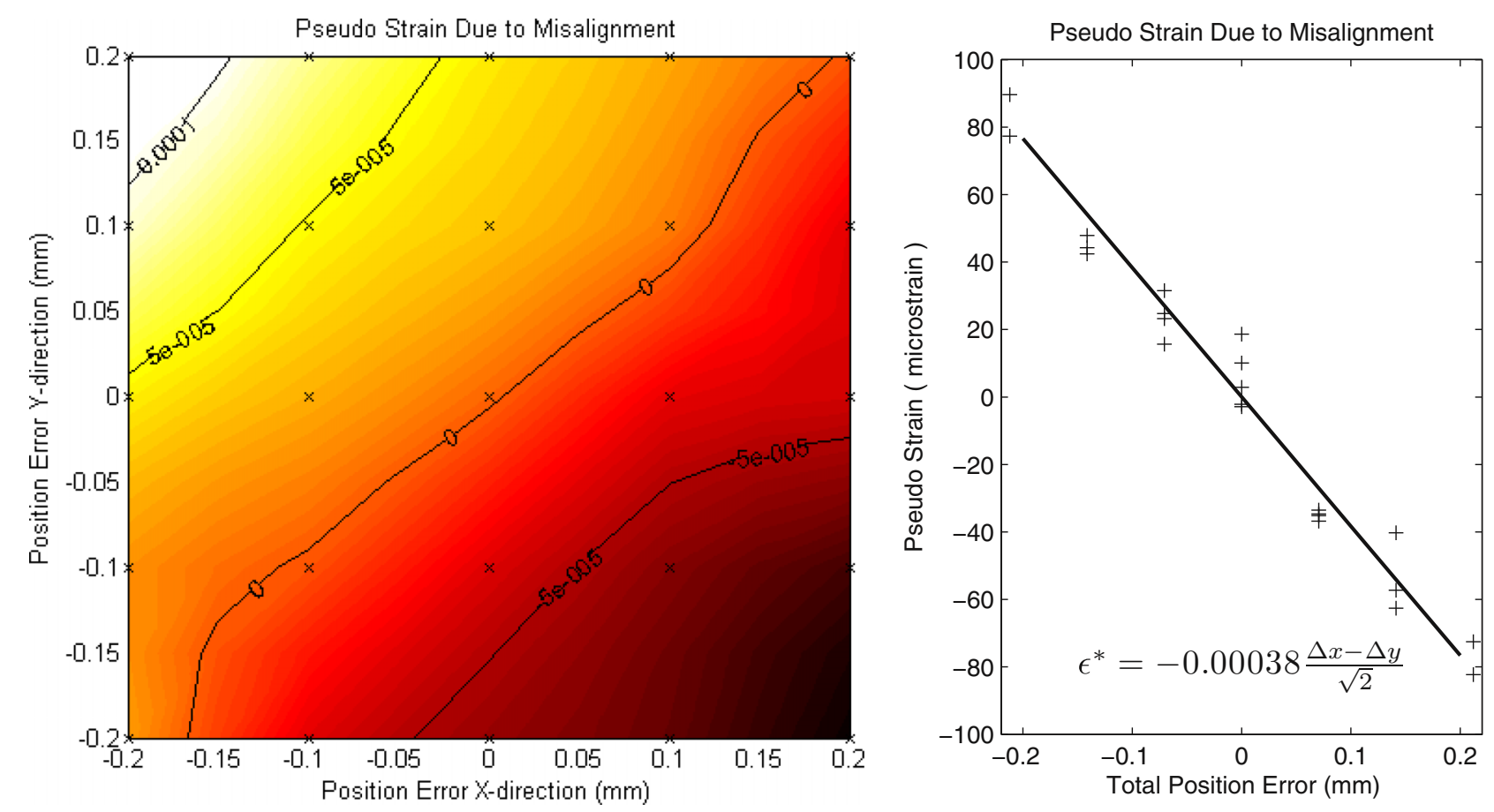

FIG. 12. (Color online) Errors observed due to positional misalignment of the particle within the gauge volume. (a) The pseudostrain error as a two-dimensional map within the horizontal plane of the instrument. (b) The same error as a function of the distance from the $x=y$ diagonal; a simple linear relationship exists in this direction which predicts around $40 \mu \epsilon$ per $100 \mu \mathrm{m}$ of misalignment in this direction.

(2) In the presence of neighbors at the same six angular positions for four different fills of the surrounding particle bed.

By comparing these two sets of measurements it was possible to gauge the magnitude of errors introduced by the combined effects of attenuation within the bed and the intrusion of neighboring particles into the gauge volume. Results of this process were as follows:

(1) The standard deviation of the six separate $d$-spacing measurements in the absence of neighbors represented an error of $12 \times 10^{-6}$ (or $12 \mu \epsilon$ ), which is well within the precision expected from the counting statistics.

(2) Over all four fills in all six positions, the standard deviation of the $d$ spacing measured in the presence of the particle bed represented an error of $72 \mu \epsilon$ (after accounting for counting and peak-fitting statistics).

This value of $72 \mu \epsilon$ represents the expected uncertainty in any strain measurement within the bed due to attenuation effects and the intrusion of neighbors into the gauge volume.

Variation between balls was then examined by making a series of measurements on three additional balls mounted on aluminium posts. In each case, the center of the ball was accurately aligned at the center of the gauge volume and a $d$ spacing was measured. Across all of these balls and the ball mounted within the die, an error representing $29 \mu \epsilon$ was observed (after accounting for counting and peak-fitting statistics).

To investigate the effects of partial illumination due to positional inaccuracy a further experiment was performed on one of the additional balls described above. This experiment consisted of scanning the center of the ball within the gauge volume over a regular three-dimensional spatial grid. Each coordinate direction was varied from -0.2 to $0.2 \mathrm{~mm}$ of positional misalignment in steps of $0.1 \mathrm{~mm}$ to arrive at 100 individual measurements. As expected from the geometry of the measurement, very little variation in $d$ spacing was observed for movement in the $z$ direction; however, a significant signal in pseudostrain was observed for deviations in the $x$ and $y$ directions. This error due to positional misalignment is shown in Fig. 12(a) in the form of a two-dimensional map in the $x-y$ plane. It is clear that a significant sensitivity exists for movement in the $x=-y$ direction within the gauge volume. Figure 12(b) shows the observed pseudostrain as a function of the absolute misalignment in this direction, yielding a linear relationship exists over the range examined. Quantitatively, the relationship predicts that an error of approximately $40 \mu \epsilon$ is to be expected for every $100 \mu \mathrm{m}$ of misalignment in this direction. The expected error for a randomly distributed misalignment (in any direction) can be calculated by averaging the absolute value of this relationship over a circular path surrounding the origin. This calculation provides for an expected error of $9 \mu \epsilon$ per $100 \mu \mathrm{m}$ if the misalignment is uniformly distributed in direction.

Using these measurements as a guide, we can estimate that each individual strain measurement made within the bed carries a normally distributed error from all sources (i.e., counting statistics, potential misalignment, attenuation, and nearest-neighbor effects) of less than around $100 \mu \epsilon$. The least-squares fitting of the final calculated strain tensor can improve this situation for certain components related to the directions of the measurements made.

The uncertainty in the individual strain components was propagated through the least-squares process and stress reconstruction by a Monte Carlo scheme. Through this process, it 
was established that the uncertainty in the axial and shear components of stress shown in Figs. 7 and 9 are 15 and $9 \mathrm{MPa}$, respectively. Unfortunately the level of uncertainty in the lateral directions was much greater. This situation could be improved by additional strain measurements within the $x-y$ plane. Given that the assembly remains intact with the same load applied, the potential for this work to continue at a later date remains.

\section{CONCLUSIONS}

We have demonstrated that neutron diffraction techniques can provide insight into the discrete nature of stress and strain within granular materials. Through a coupling of diffractionbased strain measurement techniques and neutron imaging, the full triaxial stress state within individual particles in a granular assembly can be measured. Using this approach, we have been able to directly observe inhomogeneity in the form of force chains in a real three-dimensional granular assembly.

At present the approach is only applicable to what would normally be considered very high stress states; the typical error in each strain measurement was of the order of $100 \mu \epsilon$, which simplistically corresponds to a stress level of around $20 \mathrm{MPa}$ in steel. However, through the measurement of many individual strain components and an associated least-squares process, much better precision in the final triaxial measurements are possible. It may also be possible to increase the level of precision in future by taking into account the sources and mechanisms behind the uncertainty as outlined in this work. For example, the mechanisms behind uncertainty outlined in this paper generally predict greater precision as the particle size (and associated gauge volume) is reduced; however, it should be noted that this strategy carries an associated tradeoff in the sample time required for appropriate peak-fitting statistics. Improvements in precision in the future may allow the possibility for this approach to provide information on the nature of the force network within these systems.

\section{ACKNOWLEDGMENTS}

This work was completed under the support of the Australian Research Council (Grant No. DP130104290). Access to the KOWARI diffractometer was made possible by the Bragg Institute (Program Proposal 2881). Access to the DINGO neutron imaging instrument was also made possible by the Bragg Institute through a "friendly user" arrangement during commissioning (Proposal 3071). Additional support during the experiment was provided by the Australian Institute of Nuclear Science and Engineering (AINSE).

\section{APPENDIX: SAMPLE ALIGNMENT}

Sample alignment was focused on providing appropriate coordinate transformations between the tomographic scan and the KOWARI sample stage drives. To achieve this we relied upon the measured positions of the fiducial markers (identified as $a, b, c$, and $d$ ) in the tomographic scan and the following process:
(1) The coordinates of each of the four markers in the KOWARI coordinate system were determined from a series of edge scans in a reference configuration $\left(\Omega_{e}=0\right)$.

(2) A linear mapping was then determined to transform between the position of the fiducial markers in the scan and the reference position on the instrument. This mapping was of the form

$$
\left(X_{i}-X_{i}^{a}\right)_{\Omega_{e}=0}=T_{i j}\left(x_{j}-x_{j}^{a}\right),
$$

where $\bar{x}$ and $\bar{X}$ represent positions expressed in the DINGO and KOWARI coordinate systems, respectively. This transformation can be calculated as

$$
T=M K^{-1},
$$

where $M$ and $K$ are defined as

$$
\begin{aligned}
M & =\left[\begin{array}{lll}
X_{1}^{b}-X_{1}^{a} & X_{1}^{c}-X_{1}^{a} & X_{1}^{d}-X_{1}^{a} \\
X_{2}^{b}-X_{1}^{a} & X_{2}^{c}-X_{1}^{a} & X_{2}^{d}-X_{1}^{a} \\
X_{3}^{b}-X_{1}^{a} & X_{3}^{c}-X_{1}^{a} & X_{3}^{d}-X_{1}^{a}
\end{array}\right]_{\Omega_{e}=0} \quad \text { and }, \\
K & =\left[\begin{array}{lll}
x_{1}^{b}-x_{1}^{a} & x_{1}^{c}-x_{1}^{a} & x_{1}^{d}-x_{1}^{a} \\
x_{2}^{b}-x_{1}^{a} & x_{2}^{c}-x_{1}^{a} & x_{2}^{d}-x_{1}^{a} \\
x_{3}^{b}-x_{1}^{a} & x_{3}^{c}-x_{1}^{a} & x_{3}^{d}-x_{1}^{a}
\end{array}\right]
\end{aligned}
$$

(3) The sample was then rotated to $\Omega_{e}=90^{\circ}$ and another series of edge scans was performed to find the new positions of the fiducial markers. Using a similar process to the above, a second transformation can be calculated that characterizes this movement,

$$
\left(X_{i}-X_{i}^{a}\right)_{\Omega_{e}=\frac{\pi}{2}}=\Gamma_{i j}\left(\frac{\pi}{2}\right)\left(X_{j}-X_{j}^{a}\right)_{\Omega_{e}=0},
$$

for $\psi=b, c, d$. The axis of rotation of this movement can then be determined as the eigenvector of the transformation corresponding to an eigenvalue of 1 . If we denote the normalized version of this eigenvector $\hat{R}$ [i.e., $\Gamma_{i j}\left(\frac{\pi}{2}\right) R_{j}=R_{i}$ and $R_{i} R_{i}=1$ ], we can form a transformation representing an arbitrary rotation of any angle about this axis as the following:

$$
\Gamma_{i j}\left(\Omega_{e}\right)=\delta_{i j} \cos \Omega_{e}+\sin \Omega_{e} e_{i j k} R_{k}+\left(1-\cos \Omega_{e}\right) R_{i} R_{j} .
$$

Using this transformation, we can specify any point in the original tomographic scan in the KOWARI coordinate system as

$$
\left(X_{i}\right)_{\Omega_{e}}=\left(X_{i}^{a}\right)_{\Omega_{e}}+\Gamma_{i j}\left(\Omega_{e}\right) T_{j k}\left(x_{k}-x_{k}^{a}\right) .
$$

The location of point $a$ in any given orientation can be specified by the parametric equation of a circle,

$$
\left(X_{i}^{a}\right)_{\Omega_{e}}=C_{i}+\rho_{i} \cos \Omega_{e}+e_{i j k} R_{j} \rho_{k} \sin \Omega_{e},
$$

where $\bar{C}$ is the center of the circle and $\bar{\rho}$ is a radius vector, which is orthogonal to $\hat{R} . \bar{C}$ can be uniquely determined from the position of marker $a$ in the reference $\left(\Omega_{e}=0\right)$ and rotated $\left(\Omega_{e}=90^{\circ}\right)$ orientations.

In all cases the transformations above should represent isomorphisms in the form of pure rotations. This observation can provide some additional insight on the accuracy of the particle-fitting and alignment processes. From this perspective the approach was very successful; the determinant of all 
transformations was within $1 \%$ of unity, the eigenvector of the transformation representing the rotation of the chuck, $\hat{R}$, was very closely aligned with the $y$ axis of the instrument stage, and the associated eigenvalue was 0.9990 .
As a further test, the sample was driven to $\Omega_{e}=-90^{\circ}$ and $180^{\circ}$ and spot checks were performed on a number of fiducial markers. In all cases the measured positions were within $100 \mu \mathrm{m}$ of the expected value.
[1] M. E. Fitzpatrick and A. Lodini, Analysis of Residual Stress by Diffraction Using Neutron and Synchrotron Radiation (CRC Press, Boca Raton, FL, 2003).

[2] V. Hauk, Structural and Residual Stress Analysis by Nondestructive Methods: Evaluation-Application-Assessment (Elsevier, New York, 1997).

[3] I. C. Noyan and J. B. Cohen, in Residual Stress (Springer, Berlin, 1987), pp. 117-163.

[4] E. H. Kisi and C. J. Howard, Applications of Neutron Powder Diffraction (Oxford University Press, Oxford, 2008).

[5] S. A. Hall, J. Wright, T. Pirling, E. Andò, D. J. Hughes, and G. Viggiani, Granular Matter 13, 251 (2011).

[6] C. Wensrich, E. Kisi, and V. Luzin, Granular Matter 15, 275 (2013).

[7] E. H. Kisi, C. M. Wensrich, V. Luzin, and O. Kirstein, in Materials Science Forum, Vol. 777 (Trans Tech Publications, Zurich, 2014), pp. 243-248.

[8] C. Wensrich, E. Kisi, J. Zhang, and O. Kirstein, Granular Matter 14, 671 (2012).

[9] K. L. Johnson and K. L. Johnson, Contact Mechanics (Cambridge University Press, Cambridge, 1987).

[10] J. Fleischmann, W. Drugan, and M. Plesha, J. Mech. Phys. Solids 61, 1569 (2013).

[11] C. S. Chang, S. J. Chao, and Y. Chang, Int. J. Solids Struct. 32, 1989 (1995).

[12] N. Kruyt, Int. J. Solids Struct. 51, 2336 (2014).

[13] M. Oda and H. Kazama, Geotechnique 48, 465 (1998).

[14] A. Tordesillas and M. Muthuswamy, J. Mech. Phys. Solids 57, 706 (2009).

[15] A. Tordesillas, D. M. Walker, and Q. Lin, Phys. Rev. E 81, 011302 (2010).

[16] A. Rechenmacher, S. Abedi, and O. Chupin, Geotechnique 60, 343 (2010).

[17] D. W. Howell, R. Behringer, and C. Veje, Chaos: Int. J. Nonlin. Sci. 9, 559 (1999).

[18] T. S. Majmudar and R. P. Behringer, Nature 435, 1079 (2005).

[19] D. M. Wood and D. Leśniewska, Granular Matter 13, 395 (2011).

[20] C.-h. Liu, S. R. Nagel, D. A. Schecter, S. N. Coppersmith, S. Majumdar, O. Narayan, and T. A. Witten, Science 269, 513 (1995).

[21] J. Geng, G. Reydellet, É. Clément, and R. Behringer, Physica D: Nonlin. Phenom. 182, 274 (2003).

[22] J. Zhang, Ph.D. thesis, The University of Newcastle, Australia, 2014.
[23] D. M. Mueth, H. M. Jaeger, and S. R. Nagel, Phys. Rev. E 57, 3164 (1998).

[24] G. Løvoll, K. J. Måløy, and E. G. Flekkøy, Phys. Rev. E 60, 5872 (1999).

[25] J. Brujić, S. F. Edwards, D. V. Grinev, I. Hopkinson, D. Brujić, and H. A. Makse, Faraday Discuss. 123, 207 (2003).

[26] M. Saadatfar, A. P. Sheppard, T. J. Senden, and A. J. Kabla, J. Mech. Phys. Solids 60, 55 (2012).

[27] F. Radjai, M. Jean, J.-J. Moreau, and S. Roux, Phys. Rev. Lett. 77, 274 (1996).

[28] J. F. Peters, M. Muthuswamy, J. Wibowo, and A. Tordesillas, Phys. Rev. E 72, 041307 (2005).

[29] P. T. Metzger, Phys. Rev. E 70, 051303 (2004).

[30] M. E. Cates, J. P. Wittmer, J.-P. Bouchaud, and P. Claudin, Phys. Rev. Lett. 81, 1841 (1998).

[31] S. N. Coppersmith, C.-h. Liu, S. Majumdar, O. Narayan, and T. A. Witten, Phys. Rev. E 53, 4673 (1996).

[32] C. Goldenberg and I. Goldhirsch, Phys. Rev. Lett. 89, 084302 (2002).

[33] C. Wensrich, Granular Matter 16, 597 (2014).

[34] K. Bagi, Mech. Mater. 22, 165 (1996).

[35] J. Christoffersen, M. Mehrabadi, and S. Nemat-Nasser, J. Appl. Mech. 48, 339 (1981).

[36] A. Brule and O. Kirstein, Physica B: Condensed Matter 385386, 1040 (2006).

[37] N. W. Mueggenburg, H. M. Jaeger, and S. R. Nagel, Phys. Rev. E 66, 031304 (2002).

[38] U. Garbe, T. Randall, and C. Hughes, Nucl. Instrum. Methods Phys. Res. Sec. A: Accel., Spectrom. Detect. Assoc. Equip. 651, 42 (2011).

[39] C. Wensrich, Powder Technol. 219, 118 (2012).

[40] B. P. Tighe, A. R. T. van Eerd, and T. J. H. Vlugt, Phys. Rev. Lett. 100, 238001 (2008).

[41] J. E. Andrade and C. F. Avila, Granular Matter 14, 51 (2011).

[42] R. Hurley, E. Marteau, G. Ravichandran, and J. E. Andrade, J. Mech. Phys. Solids 63, 154 (2014).

[43] X.-L. Wang, S. Spooner, and C. Hubbard, J. Appl. Crystallogr. 31, 52 (1998).

[44] S. Spooner and X.-L. Wang, J. Appl. Crystallogr. 30, 449 (1997).

[45] P. Webster, G. Mills, X. Wang, W. Kang, and T. Holden, J. Neutron Res. 3, 223 (1996). 